\title{
Silicon Nanophotonics in Data Communication, Telecommunication, Biosensing and Photovoltaics
}

\author{
Winnie Ye \\ Carleton University, Canada
}

Silicon based photonics has been under great scrutiny in recent years due to their potential for making highly compact monolithic integration of multifunctional electronic and photonic devices on the same substrate. The most popular platform is the high index contrast silicon-on- insulator (SOI) system. The high refractive index contrast between the silica cladding and the silicon waveguide core facilitates the confinement and guiding of light in structures within submicron or nanometer dimensions. In addition, the mature silicon microfabrication technology establishes a firm foundation for making low-cost and compact integrated photonic devices. A wide range of active and passive optical devices has been realized on the SOI platform. The applications of these devices can be found in high-speed communications, health industry, chemical and biological analysis, environmental monitoring, optical interconnects, and renewable energy. This talk will focus on the research related to silicon photonics at Carleton University. 